\title{
Ischemic Stroke in Patients with COVID-19 Disease: A Report of 10 Cases from Iran
}

\author{
Sanaz Ahmadi Karvigh ${ }^{a}$ Fahimeh Vahabizad ${ }^{\mathrm{a}} \quad$ Gelareh Banihashemi $^{\mathrm{a}}$ \\ Mohammad Ali Sahraian ${ }^{a}$ b Mohammad Reza Gheini $^{a} \quad$ Mahdieh Eslami $^{a}$ \\ Hoora Marhamati $^{a}$ Maryam Sadat Mirhadi ${ }^{\mathrm{a}}$ \\ aDepartment of Neurology, Sina Hospital, Tehran University of Medical Sciences, Tehran, Iran; bultiple Sclerosis \\ Research Center, Neuroscience Institute, Tehran University of Medical Science, Tehran, Iran
}

\section{Keywords}

Coronavirus · COVID-19 · Neurological manifestations - Case series study · Cerebrovascular diseases - Ischemic stroke
COVID-19 patients tend to occur as large infarct and can be seen in patients with mild to moderate pulmonary involvement.

(c) 2020 S. Karger AG, Base

\begin{abstract}
Ischemic stroke seems to be one of the most serious neurologic complications in patients with COVID-19 infection. Herein, we report a series of 10 ischemic stroke patients with concomitant COVID-19 disease. Out of 10, 8 had large infarcts ( 3 massive middle cerebral artery, 2 basilar artery, 2 posterior cerebral artery, and 1 internal carotid artery infarct territory). Two had cardiogenic embolic stroke due to atrial fibrillation. Almost half of our patients did not have a vascular risk factor. Nine did not have fever and were diagnosed with COVID-19 upon admission for stroke. Stroke occurred in the first week of respiratory symptoms with moderate pulmonary involvement. Most Patients did not have hypoxia and did not establish respiratory failure or acute respiratory distress syndrome. The blood pressures were low and hemorrhagic transformation did not occur even after antiplatelet or anticoagulant therapy. Patients had markedly increased levels of lactate dehydrogenase, C-reactive protein, and Ddimer. Three patients died. It seems that ischemic strokes in
\end{abstract}

\section{Introduction}

The evidence from SARS-CoV-2 studies implies that the virus can have central nervous system involvement [1-3]. Other reports suggest a prothrombotic state with COVID-19 [4]. Since the Coronavirus pandemic in 2020, there has been an ever-growing evidence of neurologic complications associated with COVID-19. A retrospective study form china with 214 COVID-19 patients reported 4 ischemic strokes [5]. The report suggests that the neurologic complications were seen in patients with severe COVID-19. Also, abnormalities in liver enzyme, hemoglobin, lactate dehydrogenase (LDH), d-dimer, $\mathrm{Cr}$, and lymphopenia were more common in patients with neurologic complications. Patients with COVID-19 tend to be in a prothrombotic state [4]. On the other hand, increased prothrombin time (PT), partial thromboplastin time (PTT), and decreased platelet counts is also reported karger@karger.com www.karger.com/ced

(c) 2020 S. Karger AG, Base

Karger'?
Fahimeh Vahabizad

Department of Neurology

Sina Hospital, Tehran University of Medical Sciences

Imam Khomeini Street, Tehran 009821 (Iran)

fahime.vahabizad@gmail.com 
in COVID-19 [4], which may increase the risk of hemorrhagic incidents. This is especially important in patients with cerebrovascular diseases.

Because the treatment of ischemic strokes includes different regimens of antiplatelet, anticoagulant, and thrombolytic therapies; it is important to understand the relation between COVID-19 and coagulative changes in the body. So far, a lot about the virus and its behavior is unknown. Here, we aim to describe the findings in 10 patients with ischemic stroke and COVID-19.

\section{Method}

One week after the announcement of the outbreak of COVID-19 Tehran, Iran, Sina Hospital affiliated to Tehran University of Medical Sciences became one of the official referral centers for COVID-19 on February 28, 2020. This single-center retrospective case series study was carried out based on the data extracted from the Sina registry database [6] and was approved by the Iran National Committee for ethics in biomedical research. All cases in the emergency room were screened for COVID 19 regardless of their complaints. Until April 3, 2020, we had about 508 patients admitted with moderate to severe COVID-19 diagnosis amongst which, 10 also had ischemic stroke. Due to hospital guidelines, the diagnosis of COVID-19 infection was made through clinical history and chest computed tomography (CT) scan. If highly suggestive, the diagnosis was made with the chest CT findings. If suspicious, COVID-19 PCR test was performed. Brain CT scan was performed initially for all patients with stroke symptoms and only if the brain CT scan findings were inconclusive brain magnetic resonance imaging (MRI) with diffusion-weighted imaging/apparent diffusion coefficient (DWI/ADC) map sequences were performed. We admitted patients with stroke and COVID-19 and monitored them continuously for blood oxygen saturation and need for mechanical ventilation. In the case of any neurologic symptoms' aggravation, we repeated brain CT scan (data availability: anonymized data not published within this article will be made available by request from any qualified investigator).

\section{Results}

We had 10 ischemic strokes (no intracranial hemorrhages) out of 508 COVID-19 patients (1.9\%) that correspond to 10 out of 22 (with and without COVID-19) total stroke number (45\%). We had 12 patients with stroke who did not have COVID-19 and were referred to another center after diagnosis. Table 1 shows the patients characteristics. Most of our patients aged between 50 and 90 years. Only 1 patient was 27 years old. Five had cerebrovascular disease risk factors (none had previously known atrial fibrillation $[\mathrm{AF}]$ ) and 4 were already taking anti-platelet prophylaxis prior to stroke. Most patients had experienced respiratory symptoms for $0-7$ days prior to stroke. However, only one of them had sought medical attention and was in home quarantine receiving hydroxychloroquine and azithromycin with the diagnosis of a mild COVID-19 disease. Four patients did not have any symptoms of COVID-19 and were diagnosed with the infection upon stroke admission. When present, cough and dyspnea were the most common respiratory symptoms that were aggravated at the time of admission for stroke.

In the emergency room (), only 1 patient had a $39.5^{\circ} \mathrm{C}$ fever and the vital signs were stable except for $\mathrm{pt} \# 6$ who had increase heart rate and respiratory rates ( 120 beat per minute, respiratory rate: $40 / \mathrm{min}$ ). The peripheral capillary oxygen saturation in most patients was acceptable (above 90\%). Three had peripheral capillary oxygen saturation: $88 \%$ that were intubated in the ER. Nonetheless, during the disease, 3 more needed mechanical ventilation due to decreased levels of consciousness. Of interest, the blood pressures were relatively low in our patients $(90 / 60$ $160 / 90$, median $130 / 80 \mathrm{~mm}$ of mercury [ $\mathrm{mm} \mathrm{Hg}]$ ). Two patients had irregular heart rates due to new-onset AF.

Upon admission, most of our patients were ill and had various degrees of decreased consciousness. There were no seizures nor temporary loss of consciousness. The focal neurologic deficits were severe (Table 1).

We made the diagnosis of COVID-19 infection based on chest CT scan reported by a radiologist. Most of them had bilateral moderate to severe ground glass opacities in more than 2 lobes. Two patients had mild pulmonary involvement. However, severe alveolar involvement suggestive of acute respiratory distress syndrome was not present. The brain CT scan findings showed mostly large artery involvement with large infarct size, but there was no hemorrhagic transformation in the course of the disease. Three had a malignant left middle cerebral artery (MCA) stem infarct, 2 had a top of basilar artery thrombosis, 2 right posterior cerebral artery infarct, 1 had a left internal carotid artery thromboembolic stroke, and 2 had cariogenic embolic stroke due to AF (Fig. 1).

The lab data showed that the total white blood cell count was normal to elevated $\left(6.2-18.7 \times 10^{3}\right)$ and 3 patients had lymphopenia (absolute lymphocyte count: 620, 714 , and 580). The hemoglobin levels were not dramatically decreased ranging from $12.7-15.4 \mathrm{mg} / \mathrm{dL}$. The platelet counts and Cr levels were normal (except for pt\#6 who later developed acute tubular necrosis [ATN]). PT and PTT were normal. The liver enzymes and erythrocyte sedimentation rate levels were mildly increased. All patients had elevated C-reactive protein (mean: 50.54 and SD: 48.17), D-dimer (mean: 2,746 and SD: 3,675.4), and 


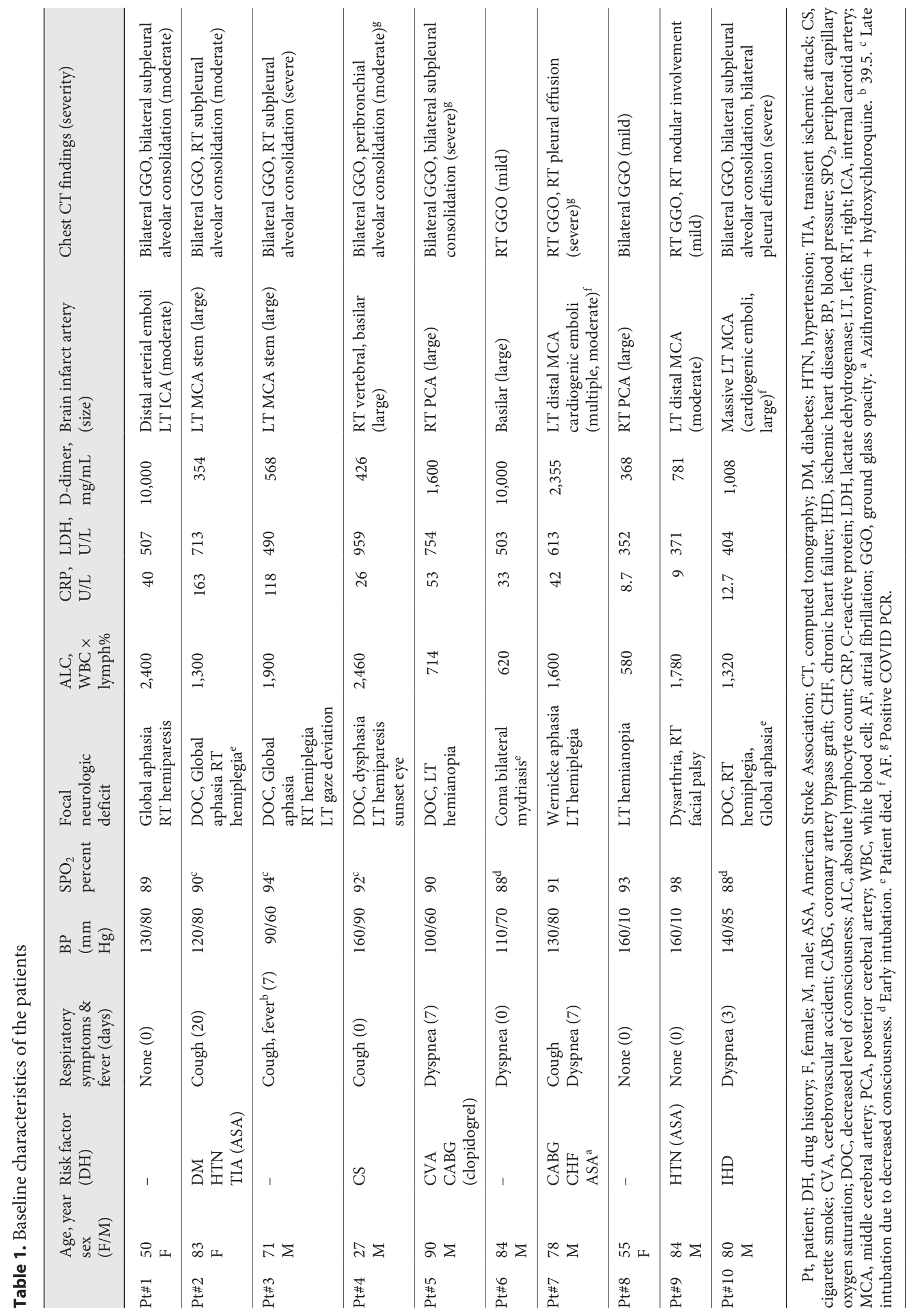



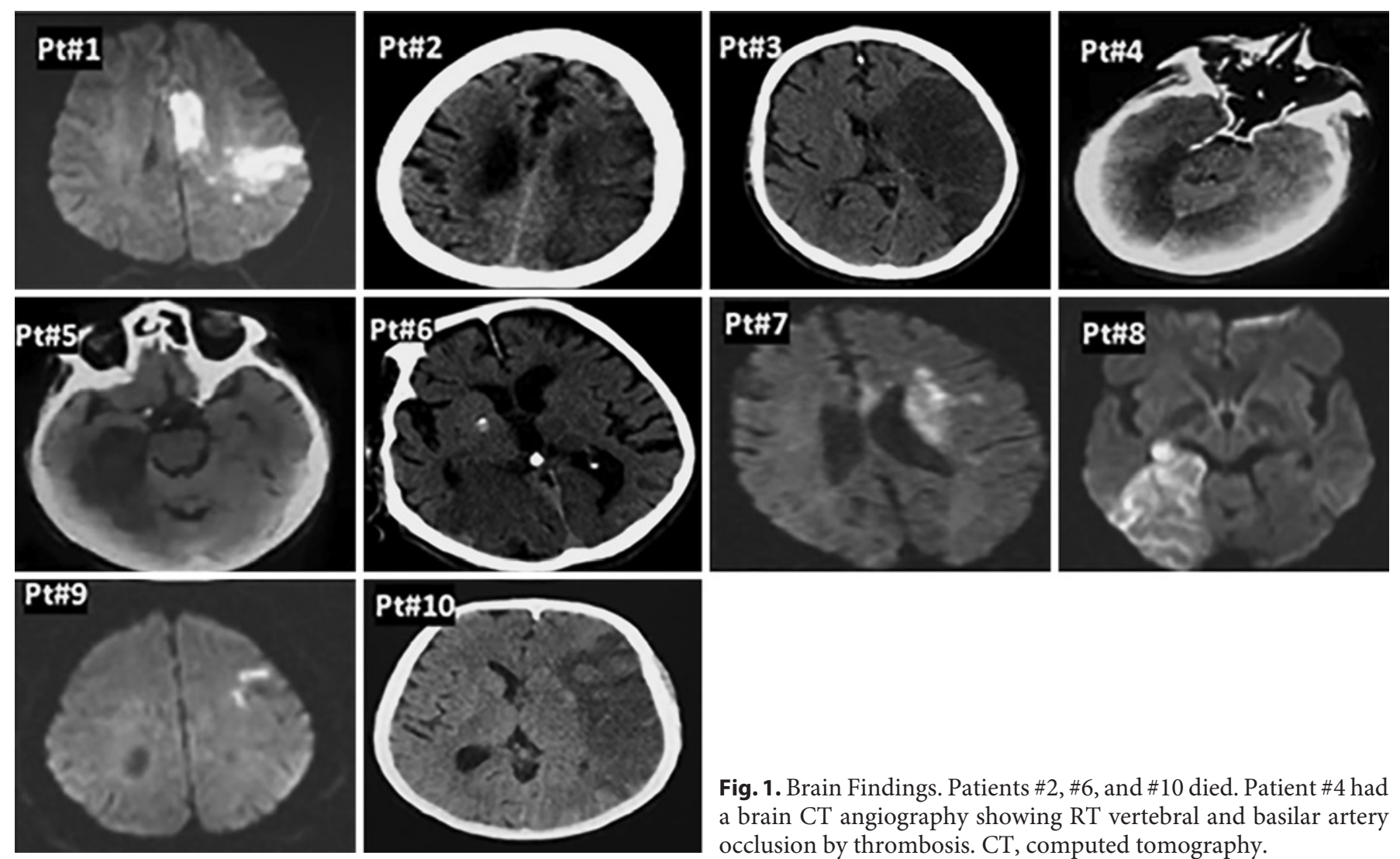

Fig. 1. Brain Findings. Patients \#2, \#6, and \#10 died. Patient \#4 had a brain CT angiography showing RT vertebral and basilar artery occlusion by thrombosis. CT, computed tomography.

LDH levels (mean: 566.6 and standard deviation [SD]: 183.5) (Table 1).

None of our patients were eligible for thrombolytic treatment. For acute ischemic stroke secondary prevention, our patients were treated based on American Heart Association/American Stroke Association guideline 2019 [7]. Seven of our patients received antiplatelet. Two patients with AF received anticoagulants after 1 week. The 27-year-old patient with extensive basilar and vertebral artery thrombosis, received a combined anti-platelet, anticoagulant treatment that resulted in dramatic improvement. Unfortunately, 3 patients died after less than a week of stroke one with malignant MCA stroke and one with top of basilar artery stroke.

\section{Discussion}

There are reports showing that thromboembolic events are increased in patients with COVID-19 [4] and apart from headache and dizziness, cerebrovascular accidents (CVAs) are amongst the common neurologic complications in this disease. It has been postulated that state of hypercoagulation along with endothelial injury following massive inflammatory response in COVID-19 could be potential contributor to developing ischemic stroke [8]. Given that CVA is also very common population-wide, it is understandable that it could be a common comorbidity when we have this high number of COVID-19 infection. Our stroke numbers are comparable to the Chinese report (1.9 vs. $1.7 \%$, respectively) [4]. The main outstanding characteristics of our stroke patients were the size of the infarct, the larger size artery involvement, and the relative absence of conventional cerebrovascular risk factors. This was noteworthy especially in our 27-year-old patient with total vertebrobasilar artery occlusion. This patient was quite an outlier. The only risk factor he possessed was smoking and curiously one can assume that prothrombotic state associated with $\mathrm{CO}$ VID-19 infection might have played an important role in his disease. The lab tests for genetic thrombophilia conditions will be performed after the anticoagulant regimen.

It appears that COVID-19 disease can be associated with an increased thrombotic state that can be associated 
with large artery thrombosis. A case series study in New York has reported 5 COVID-19 patients under 50 years with large-vessel stoke [9]. Another interesting finding was the normal to mildly elevated blood pressures in our patients. We usually anticipate a rather high blood pressure in the first day of stroke. Several mechanisms could contribute to low blood pressure in acute ischemic stroke including cardio emboli, heart failure, gastrointestinal bleeding, and sepsis [10]. In general, patients who are normotensive at the presentation of acute ischemic stroke tend to have cardio emboli source [11]. It seems that in the context of COVID-19, cardiogenic shock or sepsis could be the plausible causes of low blood pressure. In the previous reports [4], the severe neurologic complications were more common in the severe COVID-19 cases. Nevertheless, in our patients, the pulmonary involvement was mostly mild to moderate and none of our patient reached a severe respiratory distress pattern prior to stroke. Even the arterial oxygen saturations were acceptable and the late need for mechanical ventilation was due to decreased levels of consciousness. Also, in our patients the absence of fever and the fact that the COVID-19 disease was diagnosed upon admission was noteworthy. Therefore, it is of utmost importance to note that most patients were not aware of having COVID-19 disease and did not primarily complain of respiratory symptoms. In our hospital, 2 separate emergency rooms exist: 1 designated to COVID patients and the other to regular patients. Most of our patients came to the non-COVID ER. This once again emphasizes on screening all patients for COVID-19 during the epidemic since many patients may lack telltale signs of the infection such as fever. Furthermore, performing a simultaneous chest/brain CT scan seems to be a convenient method in patients with CVA.

According to previous reports, we expected abnormalities in liver enzyme, hemoglobin, $\mathrm{LDH}$, d-dimer, $\mathrm{Cr}$, and lymphopenia [5]. Similarly, we noted that the significant lab findings were abnormal such as C-reactive protein, $\mathrm{LDH}$, and D-dimer levels. But our patients did not have decreased hemoglobin, extremely low lymphocyte count or extremely high liver enzymes as was reported in a Chinese report [4]. Aside from a thrombus indicator, the Ddimer is known to be an acute phase reactant. Therefore, one can assume that the $\mathrm{D}$-dimer rise is basically due to a severe underlying COVID-19 infection. However, we were not convinced that the viral infection was severe enough to justify the rise. It would be enlightening to compare the D-dimer levels in all COVID-19 patients with and without thrombotic events. Unfortunately, the $\mathrm{D}$-dimer levels were assessed in our hospital only in pa-

Ischemic Stroke in Patients with COVID-19 Disease tients with a thromboembolic disease and we did not have the necessary data to investigate this matter. The mortality rate was high as it was expected in old age patient with massive stroke or with COVID-19 infection. There was no clear evidence of DIC or increased PT and PTT (unlike previous reports) [4] and we did not encounter a hemorrhagic transformation after antiplatelet and anticoagulant therapy. Anticoagulation with heparin has been used in critically ill patients with COVID-19. On the other hand, in acute large ischemic infarcts, there is always a hesitation of using anticoagulation for the fear of hemorrhagic transformation. One may assume that the situation is different with COVID-19 patients with stroke, but we need more reports to confirm the safety or benefit of using anticoagulants in the acute phase of stroke in these patients. Unfortunately, we did not have an eligible case for recombinant tissue plasminogen activator injection to observe the interaction of this treatment with the assumed coagulopathy of COVID-19 disease. On the other hand, large artery thrombosis may be a suitable candidate for thrombectomy which we did not perform in these cases.

There might be some possible limitations in this study. First, we did not screen patients based on their COVID PCR test. At the beginning of the COVID-19 pandemic, because of substantial number of COVID-19 patients, we did not have enough PCR tests for screening. Therefore, the initial diagnosis of COVID-19 was based on the chest CT findings. Second, this study was a retrospective study, and the patient's data were reliant on documentation in clinical histories. Third, given this study was an uncontrolled case series, there was not a comparison group to differentiate the acute ischemic stroke features in COVID-19 patients and non-COVID-19 patients.

\section{Conclusion}

In our series, about $1.9 \%$ of patients with moderate-tosevere COVID-19 had concomitant ischemic stroke. Ischemic strokes in COVID-19 patients tend to occur as large infarcts mostly due to large artery thrombosis and can be seen in patients with mild to moderate pulmonary involvement who are not aware of having COVID disease. Antiplatelet and anticoagulant therapy was not harmful in our patients, however, recombinant tissue plasminogen activator administration should be investigated in COVID-19 patients to understand the potential benefit or harm of this treatment in relation to COVID-19 coagulative/thrombotic complications.

Cerebrovasc Dis 2021;50:239-244 
We are indebted to the Research Development Canter of Sina Hospital for its support.

\section{Statement of Ethics}

Ethical approval for this study was obtained from the Iran $\mathrm{Na}$ tional Committee for ethics in biomedical research (IR.TUMS. VCR.REC.1399.400). Written informed consent for publication of this case report was obtained from the patients. If the patient died, consent was obtained from their next of kin. If the patient was vulnerable, consent was obtained from the legal guardian.

\section{Conflict of Interest Statement}

The authors have no conflicts of interest to declare.

\section{Funding Sources}

The authors received no specific funding for this work.

\section{Author Contributions}

Sanaz Ahmadi Karvigh and Fahimeh Vahabizad designed and conceptualized the study, analyzed the data, and drafted the manuscript for intellectual content. Mohammad Ali Sahraian and Mohammad Reza Gheini revised the manuscript for intellectual content. Gelareh Banihashemi, Mahdieh Eslami, Hoora Marhamati, and Maryam Sadat Mirhadi collected data. All authors discussed the results and contributed to the final manuscript.

\section{References}

1 HammingTimens IW, Bulthuis MLC, Lely AT, Navis GJ, van Goor H. Tissue distribution of ACE2 protein, the functional receptor for SARS coronavirus A first step in understanding SARS pathogenesis. J Pathol. 2004;203: 631-7.

2 Desforges M, Favreau DJ, Brison É, Desjardins J, Meessen-Pinard $\mathrm{M}$, Jacomy $\mathrm{H}$, et al. Human coronaviruses: respiratory pathogens revisited as infectious neuroinvasive, neurotropic, and neurovirulent agents; 2013.

3 Arabi YM, Balkhy HH, Hayden FG, Bouchama A, Luke T, Baillie JK, et al. Middle East respiratory syndrome. N Engl J Med. 2017; 376(6):584-94.

4 Mao L, Wang M, Chen S, He Q, Chang J, Hong C, et al. Neurological manifestations of hospitalized patients with COVID-19 in Wuhan, China: a retrospective case series study; 2020.
5 Chen T, Wu D, Chen H, Yan W, Yang D, Chen $\mathrm{G}$, et al. Clinical characteristics of 113 deceased patients with coronavirus disease 2019: retrospective study. BMJ. 2020;368: m1091.

6 Talebpour M, Hadadi A, Oraii A, Ashraf H. Rationale and Design of a Registry in a Referral and Educational Medical Center in Tehran, Iran: Sina Hospital Covid-19 Registry (SHCo-19R)

7 Warner JJ, Harrington RA, Sacco RL, Elkind MS. Guidelines for the early management of patients with acute ischemic stroke: 2019 update to the 2018 guidelines for the early management of acute ischemic stroke.
8 Hess DC, Eldahshan W, Rutkowski E. COVID-19-related stroke. Transl Stroke Res. 2020 Jun;11(3):322-5.

9 Oxley TJ, Mocco J, Majidi S, Kellner CP, Shoirah $\mathrm{H}$, Singh IP, et al. Large-vessel stroke as a presenting feature of Covid-19 in the young. N Engl J Med. 2020 May 14;382(20):e60.

10 Verschoof MA, Groot AE, Vermeij JD, Westendorp WF, van den Berg SA, Nederkoorn PJ, et al. Association between low blood pressure and clinical outcomes in patients with acute ischemic stroke. Stroke. 2020 Jan;51(1): 338-41.

11 Moores M, Yogendrakumar V, Bereznyakova O, Alesefir W, Pettem H, Stotts G, et al. Normal systolic blood pressure at presentation with acute ischemic stroke predicts cardioembolic etiology. J Am Heart Assoc. 2020 Jan 7; 9(1):e014399. 